\title{
RANGES OF DEPOSITION TEMPERATURES APPLICABLE FOR METALORGANIC VAPOR PHASE EPITAXY OF GAN FILMS VIA THE TECHNIQUE OF PENDEO- EPITAXY
}

\author{
D. B. Thomson, T. Gehrke, K. J. Linthicum, P. Rajagopal, and R. F. Davis \\ Department of Materials Science and Engineering, North Carolina State University, Box 7907, \\ Raleigh, NC 27695-7907
}

Cite this article as: MRS Internet J. Nitride Semicond. Res. 4S1, G3.37 (1999)

\begin{abstract}
Pendeo-epitaxy is a type of selective growth of thin films from the sidewalls of etched forms. The resulting films are suspended from the sidewalls and do not interface with the substrate. This process route has advantages over conventional lateral epitaxial overgrowth (LEO) techniques. In this research, pendeo-epitaxial growth of $\mathrm{GaN}$ films has been achieved on elongated $\mathrm{GaN}$ seed columns. The seed columns were etched from $\mathrm{GaN}$ grown on $6 \mathrm{H}-\mathrm{SiC}$ (0001) substrates via metalorganic vapor phase epitaxy (MOVPE). Silicon nitride mask layers atop the GaN seed columns forced growth from the sidewalls. Pendeo-epitaxial growth of GaN was investigated using several growth temperatures. Higher growth temperatures resulted in improved coalescence due to greater lateral to vertical growth ratios.
\end{abstract}

\section{INTRODUCTION}

The III-Nitride community has shown considerable interest in the technique of lateral epitaxial overgrowth (LEO) of GaN and related materials. This interest was boosted by the report of Nakamura et al [1] of a projected laser diode lifetime of 10,000 hours in GaN-based devices fabricated using LEO. In conventional lateral epitaxy, $\mathrm{GaN}$ initially grows vertically within the openings of a patterned mask layer. Lateral growth of this material from these openings and over the masked areas results when the proper process parameters are employed. Transmission electron microscopy has shown that the masked areas stop the propagation of threading dislocations which arise from lattice mismatch at the GaN/AlN and AlN/6H-SiC interfaces. As such, the overgrown $\mathrm{GaN}$ regions contain four-to-six orders of magnitude lower density of dislocations relative to the unmasked regions [2].

Conventional single layer LEO films consist of alternating lateral regions of high and low defect density GaN. Device placement requires careful alignment with respect to the underlying mask stripes to take advantage of the superior quality material. Device size is limited to the width of the mask stripes. It is therefore desirable to have a continuous layer of low defect material over the entire GaN surface such that devices can be fabricated anywhere on the wafer without confinement to particular small areas.

Nam et al [3] have obtained the desired layer via repetition of the process route used to produce the first LEO GaN layer. In this case the second set of mask stripes were placed directly over the openings of the first LEO mask. This double LEO process requires two lithography steps and two depositions of GaN films beyond the growth of the initial seed layer. The process of pendeo-epitaxy (PE) is anticipated to yield the same result--a continuous layer of low defect 
density GaN--with only one lithography step and a single growth of GaN beyond the seed layer. As its name implies, pendeo-epitaxy is the epitaxial growth of crystalline material that hangs from freestanding forms and is suspended above the substrate. In the particular PE approach used in this research, etched columnar $\mathrm{GaN}$ forms are capped with a silicon nitride mask layer. As such, pendeo-epitaxial growth of the GaN films originates only from the sidewalls of these columns. The material grows laterally and vertically until it coalesces between and over the silicon nitride masks located atop the columns in the manner employed in the conventional LEO approach. A continuous layer of low defect density GaN is thus created. This process route is capable of producing continuous layers over large areas; it is limited only by the size of the substrate.

\section{EXPERIMENTAL PROCEDURE}

Pendeo-epitaxial growth of GaN films was performed in the manner shown schematically in Figure 1.

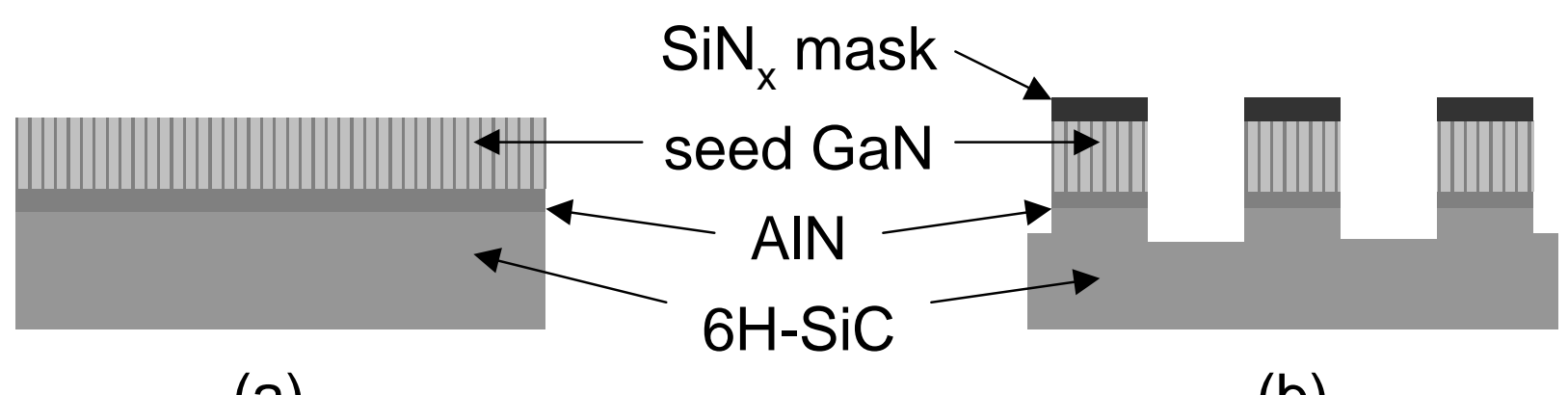

(a)

(b)

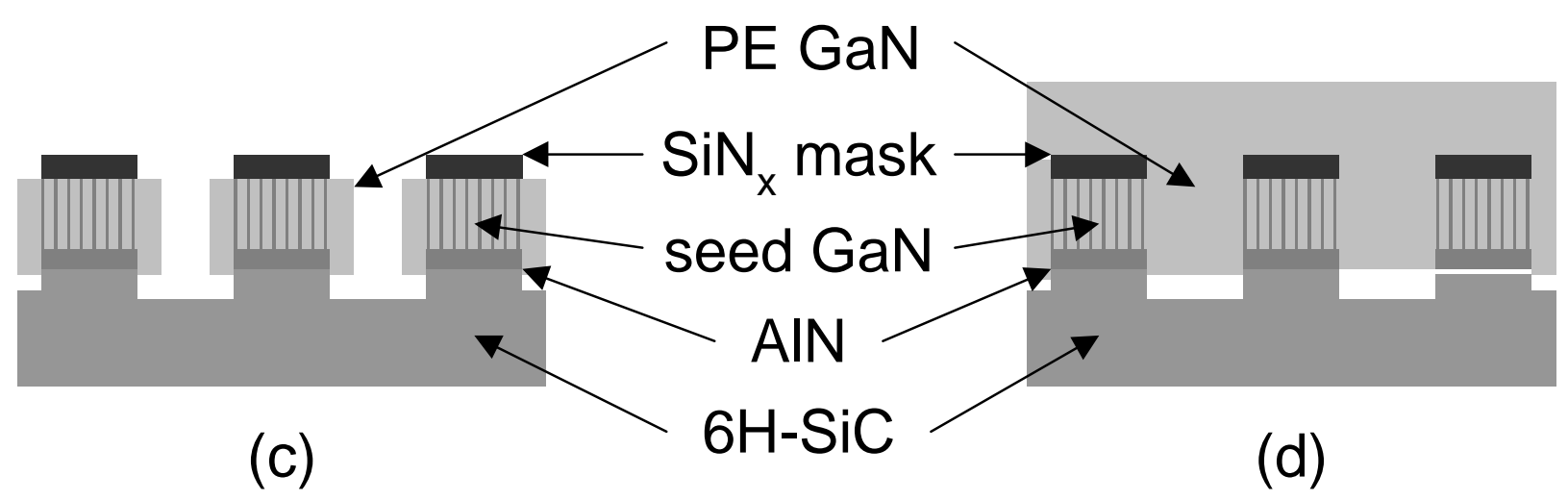

Figure 1. Schematic diagram showing the process steps for growth of pendeo-epitaxial GaN: (a) GaN seed layer, (b) etched GaN columns prior to PE growth, (c) partial growth of PE GaN showing growth only from the sidewalls, (d) coalesced growth of PE GaN.

Each substrate was prepared via growth of a $1 \mu \mathrm{m}$ thick GaN seed layer at $1000^{\circ} \mathrm{C}$ on an $\mathrm{AlN}$ buffer layer previously grown at $1100^{\circ} \mathrm{C}$ on a $6 \mathrm{H}-\mathrm{SiC}(0001)$ substrate in a cold-wall, vertical, pancake-style, RF inductively heated metalorganic vapor phase epitaxy (MOVPE) system. Additional details of the growth experiments have been previously reported [4]. The consecutive deposition of a growth mask layer of silicon nitride $\left(\mathrm{SiN}_{\mathrm{x}}\right)$ and an etch mask layer of nickel were achieved on the GaN seed layer using plasma enhanced chemical vapor deposition (PECVD) and 
e-beam evaporation, respectively. The latter mask layer was patterned using standard photolithography techniques in parallel $2 \mu \mathrm{m}$ wide stripes spaced $3 \mu \mathrm{m}$ edge-to-edge and oriented along the $[1 \overline{1} 00]$ direction of the $\mathrm{GaN}$ film. Long columns containing the GaN seed material were produced via inductively coupled plasma (ICP) etching through the $\mathrm{SiN}_{\mathrm{x}}, \mathrm{GaN}$ and $\mathrm{AlN}$ layers and into the $6 \mathrm{H}-\mathrm{SiC}$ substrate. Detailed procedures for the ICP etching have been previously reported [5]. The nickel etch mask was removed by dipping in $\mathrm{HNO}_{3}$ for approximately five minutes. The samples were subsequently cleaned by consecutive dips in trichloroethylene, acetone, methanol, and $\mathrm{HCl}$ for five minutes each and blown dry with nitrogen.

Pendeo-epitaxial growth of $\mathrm{GaN}$ from the $(11 \overline{2} 0)$ sidewalls of the columns was performed at 45 torr and temperatures ranging from $1000^{\circ} \mathrm{C}$ to $1100^{\circ} \mathrm{C}$ via MOVPE. Reactants consisting of $26 \mu \mathrm{mol} / \mathrm{min}$ triethylgallium (TEG) and $1500 \mathrm{sccm}$ ammonia were delivered into the growth chamber and entrained in $3000 \mathrm{sccm}$ of hydrogen diluent. The morphological microstructure of the PE GaN layers was characterized using scanning electron microscopy (SEM-JEOL 6400 FE) in cross-section and plan view. Surface roughness was characterized by atomic force microscopy (AFM-Digital Instruments, Inc. Dimension 3000). Additional modes of $\mathrm{PE}$ growth of $\mathrm{GaN}$ and $\mathrm{Al}_{\mathrm{x}} \mathrm{Ga}_{1-\mathrm{x}} \mathrm{N}$ films and details regarding the procedures employed have been reported externally $[6,7]$ and within this volume $[8,9]$.

\section{RESULTS AND DISCUSSION}

Partially grown $\mathrm{GaN}$ pendeo-epitaxy after 30 minutes of growth at $1000^{\circ} \mathrm{C}$ is shown in Figure 2(a).

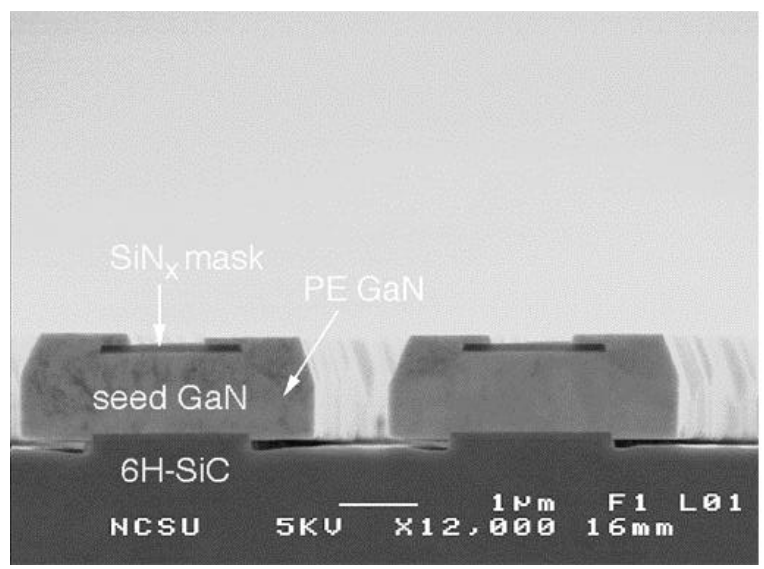

(a)

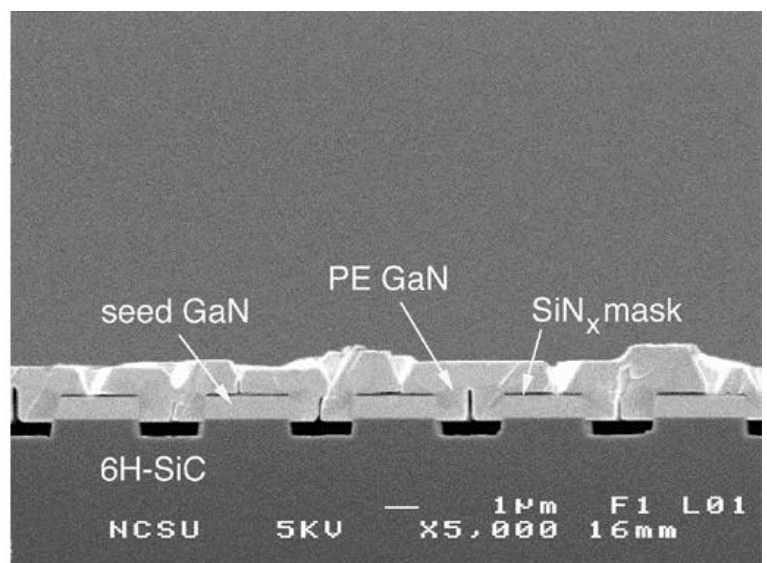

(b)

Figure 2 Cross-sectional SEM micrographs of $\mathrm{PE} \mathrm{GaN} \mathrm{grown} \mathrm{at} 1000^{\circ} \mathrm{C}$ for (a) 30 minutes, and (b) 80 minutes.

No nucleation of the GaN was observed on the surfaces of the etched $\mathrm{SiC}$ trenches. The $\mathrm{SiN}_{\mathrm{x}}$ mask forced the GaN to grow only from the sidewalls of the etched GaN columns. Growth of the $\mathrm{GaN}$ could therefore begin only in the lateral directions. As the lateral growth progressed, the $\mathrm{GaN}$ began to grow vertically once (0001) top surfaces were created. As the vertical growth reached the top of the mask, lateral growth over the mask commenced. The high lateral to vertical growth ratio (approximately three to one) caused the GaN to wrap around the mask layers. 
The results of allowing growth to continue for a total of 80 minutes at $1000^{\circ} \mathrm{C}$ are shown in Figure 2(b). Note the presence of the $\sim 60^{\circ}$ inclined $\{1 \overline{1} 01\}$ planes, which are the most stable and slowest growing planes in the GaN wurtzite crystal structure [3]. Gaps between the approaching growth fronts were also observed. These gaps suggest that the growth rates of the laterally growing faces decrease as they come into close proximity. The high vertical growth rates coupled with the continually decreasing space between converging growth fronts results in less GaN source material reaching these areas either by surface diffusion or by way of gaseous reactants.

The micrographs shown in Figure 3 demonstrate the effect of growth temperature on pendeo-epitaxial growth. Figure 3(a) is a plan view of the same film shown in Figure 2(b), which was grown for 80 minutes at $1000^{\circ} \mathrm{C}$.

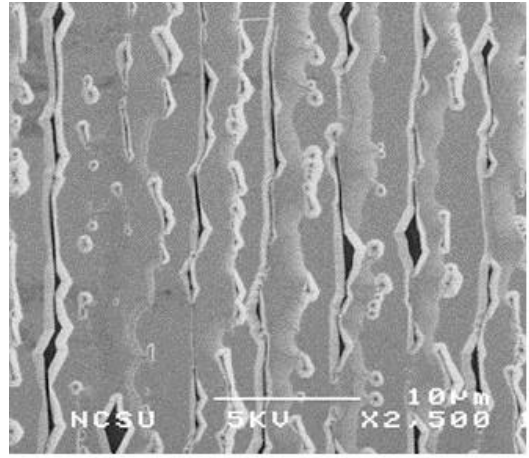

(a)

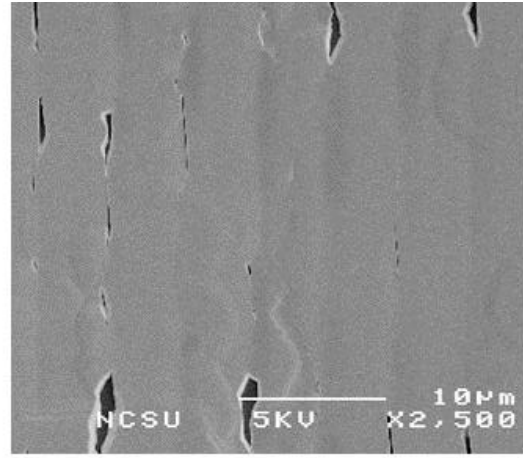

(b)

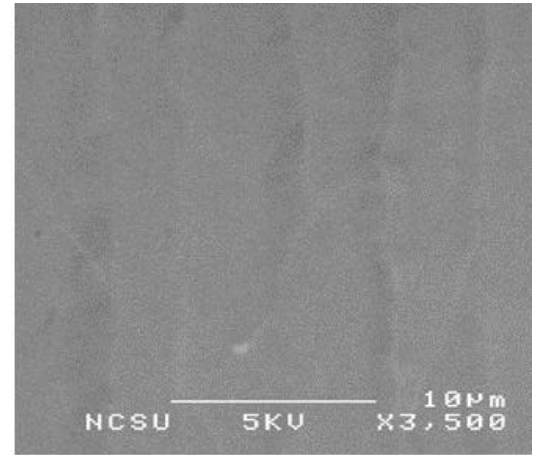

(c)

Figure 3 Plan view SEM micrographs of PE GaN grown for 80 minutes at temperatures of: (a) $1000^{\circ} \mathrm{C}$, (b) $1050^{\circ} \mathrm{C}$, and (c) $1080^{\circ} \mathrm{C}$.

Coalescence of the $\mathrm{GaN}$ is observed in only a few regions. Figure 3(b) is a plan view of PE GaN grown for 80 minutes at $1050^{\circ} \mathrm{C}$. The increase in temperature promoted the lateral growth such that the $\mathrm{GaN}$ coalesced over much of the surface. Growth for 80 minutes at a temperature of $1080^{\circ} \mathrm{C}$ resulted in a completely coalesced $\mathrm{GaN}$ surface shown in Figure 3(c). A faint periodicity consistent with the $\mathrm{SiN}_{\mathrm{x}}$ stripe spacing is observed. Characterization of this surface using AFM revealed a RMS roughness of $1.32 \mathrm{~nm}$. As the emphasis of the present study focused primarily on determination of parameters necessary for achieving coalescence, optimization of growth parameters subsequent to coalescence is expected to result in smoother PE GaN surfaces suitable for growing device structures.

A cross sectional view of coalesced $\mathrm{PE} \mathrm{GaN} \mathrm{films} \mathrm{grown} \mathrm{for} 80$ minutes at $1080^{\circ} \mathrm{C}$ between and over several columns and masks, respectively, is shown in Figure 4(a). The origins of the darker areas have not yet been determined. Figure 4(b) is a higher magnification of coalesced PE GaN. 


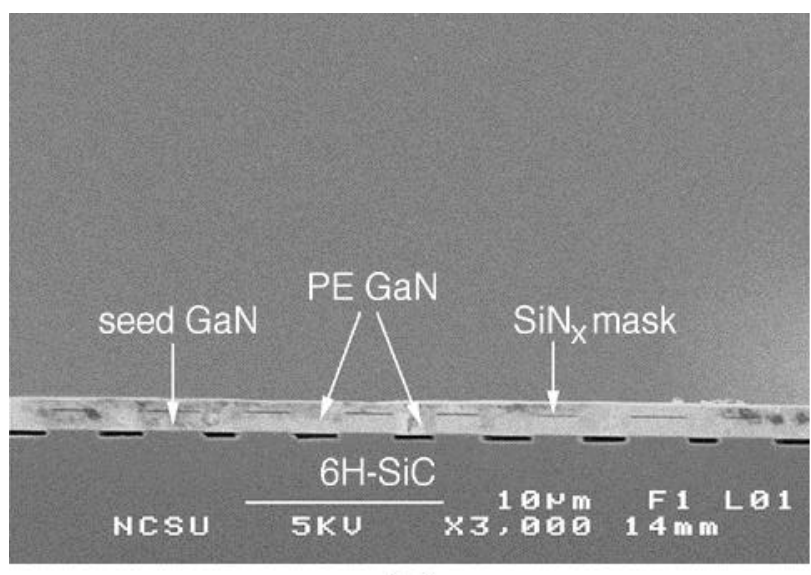

(a)

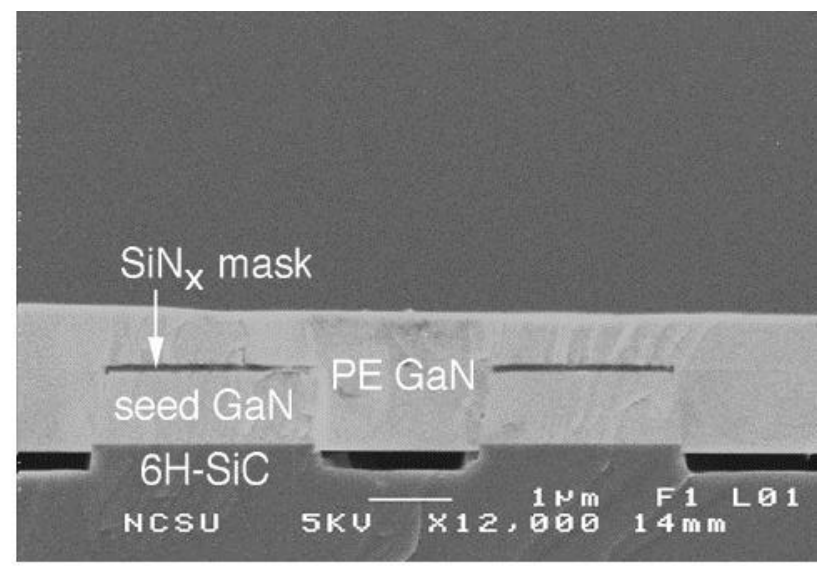

(b)

Figure 4 Cross-sectional SEM micrographs of $\mathrm{PE} \mathrm{GaN} \mathrm{grown} \mathrm{at} 1080^{\circ} \mathrm{C}$ for 80 minutes showing features at (a) low magnification and (b) high magnification.

The higher growth temperature promotes swift lateral growth until coalescence is achieved between the columns. As in the LEO approach, the GaN is observed to grow vertically over the edges of the $\mathrm{SiN}_{\mathrm{x}}$ mask stripes and laterally across the top until it coalesces with the material growing laterally from the other side of the stripe.

\section{CONCLUSIONS}

Uniform layers of $\mathrm{GaN}$ anticipated to have very low dislocation densities over the entire $\mathrm{GaN}$ surface have been grown via the technique of pendeo-epitaxy. This process route is an improvement over conventional LEO and is a more efficient method of producing the same results as multiple layers of LEO. Growth temperature was observed to have a significant effect on the morphology of the PE GaN films. Coalescence improved with increasing growth temperatures due to greater lateral to vertical growth ratios. It is expected that pendeo-epitaxy will prove itself useful for improving device quality in optoelectronic and microelectronic applications.

\section{ACKNOWLEDGMENTS}

The authors acknowledge Cree Research, Inc. for the SiC wafers. This work was supported by the Office of Naval Research under contracts \# N00014-96-1-0765 (Colin Wood, monitor) and N00014-98-1-0654 (John Zolper, monitor). 


\section{REFERENCES}

1. S. Nakamura, The $2^{\text {nd }}$ Intl. Conf. Nitride Semiconductors, Tokushima, Japan, October 25-31, (1997).

2. T. Zheleva, O.H. Nam, J.D. Griffin, M.D. Bremser and R.F. Davis, Mat. Res. Soc. Symp. Proc. 482, 393 (1998).

3. O.H. Nam, T.S. Zheleva, M.D. Bremser, D.B. Thomson and R.F. Davis, Mat. Res. Soc. Symp. Proc. 482, 301 (1998).

4. T.W. Weeks Jr., M.D. Bremser, K.S. Ailey, E.P. Carlson, W.G. Perry and R.F. Davis, Jpn. J. Appl. Phys. Lett., 67, 401 (1995).

5. S.A. Smith, C.A. Wolden, M.D. Bremser, A.D. Hanser, R.F. Davis, and W.V. Lampert, Appl. Phys. Lett. 71, 3631 (1998).

6. T.S. Zheleva, S.A. Smith, D.B. Thomson, K.J. Linthicum and R.F. Davis, J. Electr. Mat., submitted Nov. 1998.

7. K.J. Linthicum, T. Gehrke, D.B. Thomson, E.P. Carlson, P. Rajagopal, S. Smith and R.F. Davis, Appl. Phys. Lett., submitted Nov. 1998.

8. T. Gehrke, K.J. Linthicum, D.B. Thomson, P. Rajagopal, A.D. Batchelor and R.F. Davis, Mat. Res. Soc. Symp., this volume.

9. K.J. Linthicum, T. Gehrke, D.B. Thomson, K.M. Tracy, E.P. Carlson, S. Smith, T.S. Zheleva and R.F. Davis, Mat. Res. Soc. Symp., this volume. 